\title{
Threshold $\pi^{-}$production on the deuteron
}

\author{
B. Strandberg ${ }^{1, \star}$, J. R. M. Annand ${ }^{1}$, W. Briscoe ${ }^{2}$, G. Feldman², K. Fissum ${ }^{3}$, K. Hansen ${ }^{4}$, \\ L. Isaksson ${ }^{4}$, L. Myers ${ }^{5}$, and G. V. O'Reilly ${ }^{6}$ \\ for the MAXTagg@Lund Collaboration \\ ${ }^{1}$ The University Of Glasgow, Glasgow, United Kingdom \\ ${ }^{2}$ The George Washington University, Washington, D.C., USA \\ ${ }^{3}$ Lund University, Lund, Sweden \\ ${ }^{4}$ MAX IV Laboratory, Lund University, Lund, Sweden \\ ${ }^{5}$ Bluffton University, Bluffton, USA \\ ${ }^{6}$ University of Massachusetts Dartmouth, Dartmouth, USA
}

\begin{abstract}
An experiment that aims to measure the $\pi^{-}$photoproduction cross-section $\sigma(E)$ on the deuteron $\gamma+{ }^{2} \mathrm{H} \rightarrow \pi^{-}+2 \mathrm{p}$ near threshold is discussed. The main concepts of the experimental technique are presented alongside some preliminary signals.
\end{abstract}

\section{Introduction}

Pion photoproduction on the nucleus is described by various theoretical frameworks and experimental data is vital to test the accuracy of the models. Numerous experiments have been performed to study the $\pi^{0}$ channel, but data on the charged channels is scarce. To the best of the authors knowledge no data exists for $\pi^{-}$photoproduction below incident photon energy of $158 \mathrm{MeV}$. In this proceedings paper an experiment that aims to measure the $\pi^{-}$photoproduction

$$
\gamma+{ }^{2} \mathrm{H} \rightarrow \pi^{-}+2 \mathrm{p}
$$

cross-section on the deuteron from threshold to $E_{\gamma}=160 \mathrm{MeV}$ is discussed. Sect. 2 outlines the basics of the experimental setup. Sect. 3 is dedicated to our event counting technique and Sect. 4 will present some aspects of the current analysis status.

\section{Experimental setup}

The experiment was performed at MAXLab in Lund, Sweden. The MAXLab facility provided for the experiment a pulse-stretched electron beam with an energy of $\sim 190 \mathrm{MeV}$. The bremsstrahlungtagging technique $[1,2]$ was used to convert the electron beam into a tagged photon beam with an energy range from $140-160 \mathrm{MeV}$. The tagged photon beam was directed on a liquid deuterium target. Three large Sodium-Iodide (NaI) crystal detectors (named BUNI [3], CATS [4] and DIANA [5]) were set up at three theta angles $\theta=60^{\circ}, \theta=120^{\circ}$ and $\theta=150^{\circ}$ to detect photons originating from the liquid deuterium target volume. The floor plan of the experiment is depicted in Fig. 1.

\footnotetext{
^e-mail: b.strandberg.1@ research.gla.ac.uk
} 


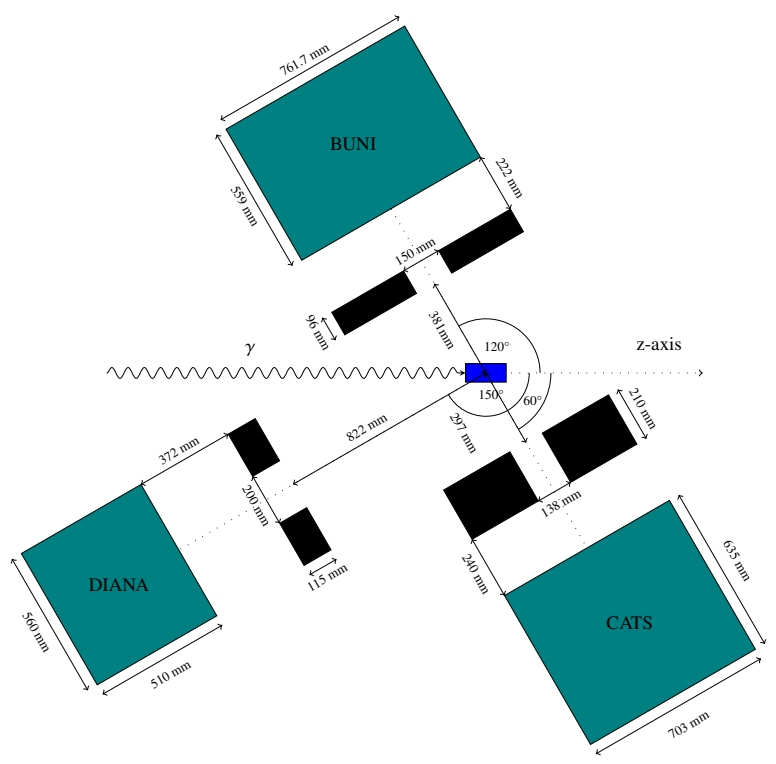

Figure 1. A drawing of the experimental setup in Lund, Sweden. The green-blue colour indicates the $\mathrm{NaI}$ detector crystals, the black colour depicts the front collimators and the blue colour represents the liquid deuterium target. There were also thin veto paddles in front of each collimator to veto charged particles (not shown).

\section{Event counting}

As the pions in reaction (1) are created near threshold their kinetic energy is low and with a high probability they are captured on the deuteron. The capture can go through two dominant channels

$$
\begin{aligned}
& \pi^{-}+{ }^{2} \mathrm{H} \rightarrow \gamma+2 \mathrm{n}, \\
& \pi^{-}+{ }^{2} \mathrm{H} \rightarrow 2 \mathrm{n} .
\end{aligned}
$$

For our analysis we are especially interested in the radiative capture reaction Eq. (2). The energy spectrum of the emitted photon has a distinct peak at $\sim 130 \mathrm{MeV}$ and its shape is well known from before [6-8]. Our plan is to count the $\pi^{-}$that have been produced on the deuteron by counting the $\pi^{-}$ that are captured on the deuteron. For this method to succeed one needs to know:

1. The percentage of $\pi^{-}$that are captured.

2. The branching ratio of non-radiative capture (Eq. (3)) to radiative capture (Eq. (2)).

3. The percentage of $\pi^{-}$that undergo some other process - decay, charge exchange, pair production or double-radiative capture.

We have successfully used the Geant4 [10] simulation package to assess how many $\pi^{-}$are captured and how many decay. The branching ratio of reaction (3) relative to (2) has been measured by Highland to be $2.83 \pm 0.04$ [9]. All other processes have a total probability of less that $1 \%$ [11-13]. Therefore the information to estimate the number of produced $\pi^{-}$using the measured number of radiative captures is available.

\section{Analysis}

\subsection{Dominant backgrounds}

Multiple background sources contaminate the photon spectrum of reaction (2). There are two channels that produce energetic neutrons which can mimic the detection of a photon, reaction (3) and the 
photodisintegration reaction

$$
\gamma+{ }^{2} \mathrm{H} \rightarrow \mathrm{n}+\mathrm{p}
$$

The preliminary simulations of neutron backgrounds and the capture photon spectrum are shown in Fig. 2. However, the $\pi^{-}$capture occurs almost instantaneously and the capture $\gamma$ 's are separable from the neutron backgrounds by analysing the relative arrival times of the event triggers.

In addition to the neutron contamination there are backgrounds from $\pi^{0}$ production and from the Kapton vessel holding the liquid deuterium. The background from Kapton can come from various processes and would thus be difficult to simulate, therefore an empty vessel run was taken to estimate Kapton background contributions. The simulation of the $\pi^{0}$ background alongside the measured Kapton background (preliminary) is shown in Fig. 3. In overall the background contamination sources are well understood, but the analysis has not yet been finalised.

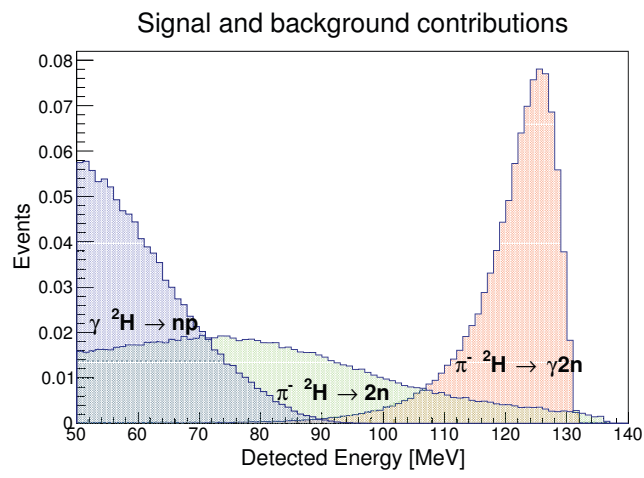

Figure 2. Photon spectrum model of reaction (2) and the neutron background simulations of reactions (3), (4).

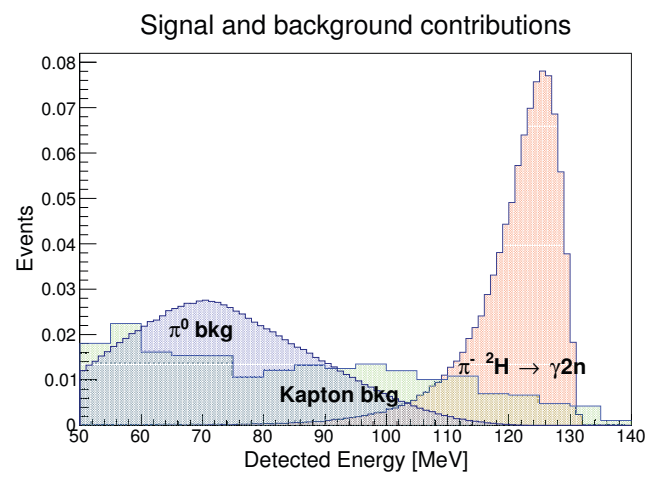

Figure 3. Photon spectrum model of reaction (2), the $\pi^{0}$ background model and the Kapton background.

\subsection{Preliminary signals}

The preliminary signals in the CATS detector are displayed in Fig. 4. The data has been binned with respect to the incident photon energy to eight bins from $140-160 \mathrm{MeV}$. Some of the neutron background has been successfully separated from the photon signal, but all background has not yet been accounted for. The radiative capture spectrum can be seen to grow significantly with increasing incident photon energy, indicating the increased production of $\pi^{-}$. The yield is dropping in the highest energy bins, but this is due to a reduced tagged photon flux and an increasing number of $\pi^{-}$exiting the target volume without being captured. These preliminary results suggest that it will be possible to extract a $\pi^{-}$photoproduction cross section.

\section{Summary}

We have provided an overview of an experiment that aims to measure the cross-section $\sigma(E)$ of the reaction $\gamma+{ }^{2} \mathrm{H} \rightarrow \pi^{-}+2 \mathrm{p}$ near the threshold region. A brief summary of the experimental setup and event counting has been presented. In the current status of the analysis the main backgrounds have been identified and the preliminary signals have been extracted.

This work was supported by the United Kingdom's Science and Technology Facilities Council (STFC) from grant number ST/L005719/1. B. Strandberg acknowledges support from The Scottish Universities Physics Alliance (SUPA). 


\section{Preliminary results in CATS detector}

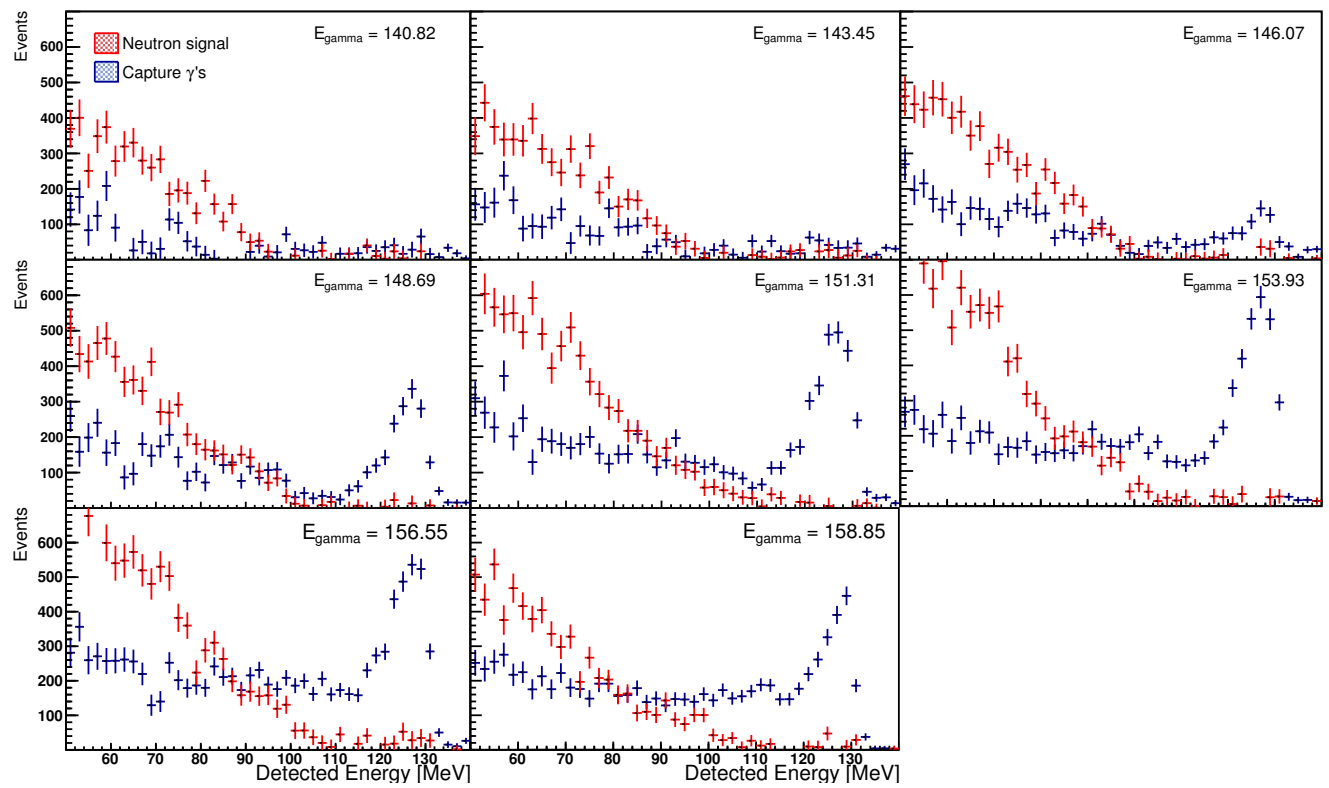

Figure 4. Signals in CATS detector. The photon signal (blue) from the capture reaction (2), the neutron background (red) from reactions (2), (4).

\section{References}

[1] J-O. Adler et al., Nucl. Instrum. Meth. A 294, 15 (1990)

[2] J-O. Adler et al., Nucl. Instrum. Meth. A 388, 17 (1997)

[3] J. P. Miller et al., Nucl. Instrum. Meth. A 270, 431 (1988)

[4] A. Hünger et al., Nucl. Phys. A 620, 385 (1997)

[5] L. S. Myers, Ph.D. thesis, University of Illinois at Urbana-Champaign, Champaign, IL, USA, 2010

[6] B. Gabioud et al., Phys. Rev. Lett. 42, 1508 (1979)

[7] G. F. de Téramond et al., Phys. Rev. C 36, 691 (1987)

[8] W. Gibbs et al., Phys. Rev. C 11, 90 (1975)

[9] V. L. Highland et al., Nucl. Phys. A 365, 333 (1981)

[10] J. Allison et al., IEEE Trans. Nucl. Sci. 53, 270 (2006)

[11] S. Tripathi et al., Phys. Rev. C 75, 064603 (2007)

[12] R. MacDonald et al., Phys. Rev. Lett. 38, 746 (1977)

[13] D. W. Joseph et al., Phys. Rev. 119, 805 (1960) 